\title{
Optimistic Fair Exchange Based on Publicly Verifiable Secret Sharing
}

\author{
Gildas Avoine and Serge Vaudenay \\ EPFL \\ http://lasecwww.epfl.ch
}

\begin{abstract}
In this paper we propose an optimistic two-party fair exchange protocol which does not rely on a centralized trusted third party. Instead, the fairness of the protocol relies on the honesty of part of the neighbor participants. This new concept, which is based on a generic verifiable secret sharing scheme, is particularly relevant in networks where centralized authority can neither be used on-line nor off-line.
\end{abstract}

\section{Introduction}

A two-party fair exchange protocol is a protocol in which two participants, an originator $P_{o}$ and a recipient $P_{r}$, wish to exchange items $m_{o}$ and $m_{r}$ in a fair way, i.e. such that no party can gain any advantage by cheating. There are two major kinds of two-party fair exchange protocols: those which rely on a Trusted Third Party (TTP) to ensure the fairness of the protocol, and those which do not. Even and Yacobi proved however in 1980 [9] that it is impossible to ensure a perfect fairness between only two participants without using a TTP. Protocols without TTP (e.g. $[3,5,7]$ ) are therefore only able to ensure fairness in a gradual or probabilistic way. The probability of fairness increases with the number of messages which are exchanged between the two participants. This implies a communication complexity cost which is too important for most of the practical applications. Finally, these protocols usually require that the involved parties have roughly equivalent computational powers, an assumption which is difficult to guarantee when dealing with heterogeneous networks. The second category of protocols rely on the use of a TTP to guarantee the fairness of the protocol. In all protocols the TTP acts as a central authority. TTP can be on-line, i.e. it is required in every exchange, or off-line (e.g. $[1,2,11,15])$. In this latter case, Asokan, Schunter, and Waidner invented the notion of optimistic protocol [1], where TTP is required only in case of conflict among participants. Fairness is ensured in a deterministic way and with few exchanges, but items must be either revocable or generatable by the TTP. Unfortunately, many environments, such as mobile ad hoc networks (MANET), do not allow either the use of a centralized authority (even in an optimistic case) for topologic reason, nor the usage of gradual protocols, which generate huge communication overheads. It is therefore important to design protocols based on other concepts. We propose a protocol which relies on the honesty of some "neighbor participants": when the exchange 
runs well, no communication overhead is required to any third party, but when a conflict occurs, neighbor participants are requested to restore fairness, by recovering the unreceived item. The recovery protocol relies on a publicly verifiable secret sharing scheme (PVSS). Indeed, a secret sharing scheme allows to share a secret among participants such that only certain subsets of participants can recover the secret. When the scheme is publicly verifiable, anybody is able to check whether the distributed shares are correct.

In what follows we define the protocol requirements, and the communication and security model. In Section 2, we recall briefly the verifiable secret sharing concept and describe such a practical protocol. We propose then our optimistic two-party fair exchange protocol based on a generic PVSS. A security analysis is finally provided in Section 4 .

\section{Communication and Security Model}

\subsection{Requirements}

Several (different) definitions for the fair exchange are available in the literature; most of them are context-dependent. We use the following common definition.

Definition 1 (exchange protocol). An exchange protocol between an originator $P_{o}$ and a recipient $P_{r}$ is a protocol in which $P_{o}$ and $P_{r}$ own some items $m_{o}$ and $m_{r}$ respectively and aim at exchanging them. We define the security properties as follows.

1. Completeness: when there is no malicious misbehavior, $P_{o}$ gets $m_{r}$ and $P_{r}$ gets $m_{o}$ at the end of the protocol.

2. Fairness: when at least one of the two participants follows the protocol, the exchange terminates so that either $P_{o}$ gets $m_{r}$ and $P_{r}$ gets $m_{o}$ (success termination), or $P_{o}$ gets no information about $m_{r}$ and $P_{r}$ gets no information about $m_{o}$ (failure termination).

3. Timeliness: the exchange eventually terminates.

4. Privacy: no other participant gets any information about $m_{o}$ and $m_{r}$.

5. Optimism: no other participant is involved when $P_{o}$ and $P_{r}$ are honest.

We say that the protocol is a fair exchange protocol when it is complete, fair, and timely.

According to this definition, we design in this paper an optimistic fair exchange protocol between two parties, that do not require centralized TTP, implies reasonably low communication overhead, and protects privacy.

\section{$2.2 \quad$ Threat Model}

We say that an active participant is honest if he follows the protocol; otherwise he is dishonest. Note that due to the communication assumptions (below), all messages from honest participants are eventually delivered. The fairness of the 
protocol is ensured when either $P_{o}$ or $P_{r}$ is dishonest. Note that we do not need to consider the case where both $P_{o}$ and $P_{r}$ are dishonest: in this case they obviously always have the ability to halt the exchange on an unfair termination. We now consider the passive participants' behaviors.

- $\mathcal{B}_{1}$ : participants who honestly collaborate with both $P_{o}$ and $P_{r}$.

- $\mathcal{B}_{2}$ : participants who may harm $P_{o}$ by colluding with $P_{r}$.

- $\mathcal{B}_{3}$ : participants who may harm $P_{r}$ by colluding with $P_{o}$.

- $\mathcal{B}_{4}$ : participants who do not collaborate at all.

Note that $\mathcal{B}_{1}, \mathcal{B}_{2}, \mathcal{B}_{3}$, and $\mathcal{B}_{4}$ form a partition of the passive participants $\mathcal{P}$. We denote $b_{i}=\left|\mathcal{B}_{i}\right|$ where $1 \leq i \leq 4$. We assume that participants cannot move from one set to another, focusing on the "honesty status" at the time of the recovery protocol only.

\subsection{Communication Model and Hypothesis}

Definition 2 (secure channel). A channel is said to be secure if it ensures confidentiality, integrity, authentication, sequentiality, and timeliness.

Confidentiality ensures that the message is kept secret from any third party. Integrity ensures that the message cannot be modified by any third party. Authentication ensures that no third party can insert a forged message in the channel. Sequentiality ensures that the sequence of messages received by one party is equal to the sequence of messages sent by the other party in the same ordering at some time. Timeliness ensures that a message inserted into the channel is eventually delivered.

Remark 1. Sequentiality ensures that no messages are swapped, dropped, or replayed.

Definition 3 (environment). We say that two entities $P$ and $P^{\prime}$ are in the same environment if and only if $P$ and $P^{\prime}$ are able to communicate through a secure channel. We let Env $v_{P}$ denote the set of all the entities which are in the same environment as $P$

Remark 2. The relation $P^{\prime} \in E n v_{P}$ between $P$ and $P^{\prime}$ is symmetric but not transitive due to the timeliness requirement.

Definition 4 (participant). We say that an entity which is involved in the protocol, either during the exchange stage or the recovery stage, is a participant. Participants which are only involved in the recovery stage are said passive; otherwise they are said active.

Remark 3. In an optimistic two-party fair exchange, only participants $P_{o}$ and $P_{r}$ are active.

Hypothesis 1. In what follows we assume that $P_{r} \in E_{n v_{P_{o}}} ; P_{r}$ knows a subset of passive participants $\mathcal{P}$ of $E n v_{P_{o}} \cap E n v_{P_{r}}$ and a constant $T_{\max }<+\infty$ such that 
messages from $P_{r}$ to any participant in $\mathcal{P}$ are always delivered within a time delay less than $T_{\max } ; b_{1}>0$; and $P_{o}$ and $P_{r}$ know some constant $k$ such that $b_{2}<k \leq b_{2}+b_{1}$.

We give here two examples in order to illustrate this assumption.

Example 1. If $P_{o}$ and $P_{r}$ know that there is a majority of honest participants in the network i.e. $b_{1}>\frac{n}{2}$ then we take $k=\left\lceil\frac{n}{2}\right\rceil$.

Example 2. If $P_{o}$ knows that at least $40 \%$ of the network is honest with him (i.e. $b_{1}+b_{3} \geq \frac{2 n}{5}$ ) and $P_{r}$ knows that at least $70 \%$ of the network is honest with him (i.e. $b_{1}+b_{2} \geq \frac{7 n}{10}$ ) then we can take $k$ such that $\left\lfloor\frac{6 n}{10}\right\rfloor<k \leq\left\lceil\frac{7 n}{10}\right\rceil$. For instance, if $n=100, k$ is chosen such that $60<k \leq 70$. We show in Section 3 that $k$ is actually the threshold of the secret sharing.

\section{Optimistic Two-Party Fair Exchange Protocol}

In this section, we first recall the notion of publicly verifiable secret-sharing; then we give an optimistic two-party fair exchange protocol. The main idea consists of sharing items among $n$ participants such as $k$ participants are enough to recover these items in case of conflict. The constraints on $k$ will be analyzed in Section 4.

\subsection{Publicly Verifiable Secret Sharing}

Secret sharing $[4,13]$ allows to share a secret $m$ among several participants such that only some specific subsets of participants can recover $m$ by collusion. In the Shamir secret sharing scheme, there is a threshold $k$ so that only subsets of at least $k$ participants can reconstruct $m$. A drawback of the Shamir scheme is that participants cannot verify that the distributed shares effectively allow to recover the secret $m$. In other words, the basic secret sharing scheme assumes that the dealer is not malicious. Verifiable secret sharing $[6,10,12,14]$ resists to a malicious dealer who sends wrong shares: each participant can indeed check his own share. In Publicly verifiable secret sharing [12,14], introduced by Stadler in 1996, anybody can perform this verification and not only the participants. Below we describe a model for non-interactive publicly verifiable secret sharing (PVSS).

Distribution stage. The dealer generates the shares $m_{i}$ of $m$ and then publishes the encrypted values $E_{i}\left(m_{i}\right)$ such that only the participant $P_{i}$ is able to decrypt $E_{i}\left(m_{i}\right)$. The dealer also publishes an information $\Delta$ containing $\theta=\mathcal{W}(m)$ where $\mathcal{W}$ is a one-way function. This information allows to prove that the distributed shares are correct i.e. they allow to recover some $m$ such that $\mathcal{W}(m)=\theta$.

Verification stage. Given the $P_{i}$ s' public keys, the $E_{i}\left(m_{i}\right) \mathrm{s}, \Delta$, and a verification algorithm, anybody can verify that the shares allow to recover some $m$ such that $\mathcal{W}(m)=\theta$.

Reconstruction stage. The participants decrypt their share $m_{i}$ from $E_{i}\left(m_{i}\right)$ and pool them in order to recover $m$. 


\subsection{A Practical Publicly Verifiable Secret Sharing Scheme}

We describe in this section a practical publicly verifiable secret sharing scheme which has been proposed by Stadler in [14]. This scheme relies on both ElGamal's public key cryptosystem [8] and on the double discrete logarithms assumption [14]. Let $p$ be a large prime number so that $q=(p-1) / 2$ is also prime, and let $h \in(\mathbb{Z} / p \mathbb{Z})^{*}$ be an element of order $q$. Let $G$ be a group of order $p$, and let $g$ be a generator of $G$ such that computing discrete logarithms to the base $g$ is difficult. Let $m \in \mathbb{Z} / p \mathbb{Z}$ be the secret and let $\mathcal{W}(m)=g^{m}$. As in Shamir's scheme, we assume that a publicly known element $x_{i} \in \mathbb{Z} / p \mathbb{Z}, x_{i} \neq 0$, is assigned to each participant $P_{i}$. We assume also that each participant $P_{i}$ owns a secret key $z_{i} \in \mathbb{Z} / q \mathbb{Z}$ and the corresponding public key $y_{i}=h^{z_{i}} \bmod p$.

Distribution stage. The dealer chooses random elements $a_{j} \in \mathbb{Z} / p \mathbb{Z}(j=$ $1, \ldots, k-1)$ and publishes the values $A_{j}=g^{a_{j}}(j=1, \ldots, k-1)$ in $\Delta$. Then he securely computes the share

$$
m_{i}=m+\sum_{j=1}^{k-1} a_{j} x_{i}^{j} \bmod p
$$

for $P_{i}$ and he publishes the value $g^{m_{i}}$ in $\Delta(1 \leq i \leq n)$. He uses the ElGamal encryption: he chooses a random value $\alpha_{i} \in \mathbb{Z} / q \mathbb{Z}$, computes the pair

$$
E_{i}\left(m_{i}\right)=\left(\sigma_{i}^{1}, \sigma_{i}^{2}\right)=\left(h^{\alpha_{i}}, m_{i}^{-1} \cdot y_{i}^{\alpha_{i}}\right) \bmod p
$$

and publishes it in $\Delta(1 \leq i \leq n)$. The precise content of $\Delta$ is described below.

Verification stage. The first step of this procedure consists in verifying the consistency of the shares. Anybody is able to perform this step by checking whether $g^{m_{i}}=g^{m} \cdot \prod_{j=1}^{k-1} A_{j}^{x_{i}^{j}}$, obtained by exponentiating (1), is satisfied in $G$ (Note that $\Delta$ includes $g^{m}, g^{m_{i}}, A_{j}$ ).

The second step consists in verifying that the pairs $\left(\sigma_{i}^{1}, \sigma_{i}^{2}\right)$ really encrypt the discrete logarithms of public elements $g^{m_{i}}$. This verification is based on the fact that the discrete logarithm of $\sigma_{i}^{1}=h^{\alpha_{i}}$ to the base $h$ equals the double discrete logarithm of $g^{m_{i} \sigma_{i}^{2}}=g^{\left(y_{i}^{\alpha_{i}}\right)}$ to the bases $g$ and $y_{i}$. One may give a zeroknowledge interactive verification procedure between the dealer and participants as described on Fig. 1; we describe here the non-interactive version which is obtained by simulating the verifier by a hash function. We assume that the dealer randomly picked some values $w_{i, \ell} \in \mathbb{Z} / q \mathbb{Z}(1 \leq \ell \leq L$ where $L \approx 100$ from [14]) for each share $m_{i}$ and computed:

$$
\begin{aligned}
\delta_{i, \ell} & :=h^{w_{i, \ell}} \bmod p \\
\gamma_{i, \ell} & :=g^{y_{i}{ }_{i, \ell}} \\
r_{i, \ell} & :=w_{i, \ell}-c_{i, \ell} \alpha_{i} \bmod q
\end{aligned}
$$


where $c_{i, \ell}$ denotes the $\ell$-th bit of $c_{i}=\mathcal{H}\left(g^{m_{i}}\left\|\sigma_{i}^{1}\right\| \sigma_{i}^{2}\left\|\delta_{i, 1}\right\| \gamma_{i, 1}\|\ldots\| \delta_{i, L} \| \gamma_{i, L}\right)$ with $\mathcal{H}$ a hash function from $\{0,1\}^{*}$ to $\{0,1\}^{L}$. Participants have therefore to check whether $\delta_{i, \ell}=h^{r_{i, \ell}} \sigma_{i}^{1 c_{i, \ell}} \bmod p$ and $\gamma_{i, \ell}=\left(g^{1-c_{i, \ell}} g^{m_{i} c_{i, \ell} \sigma_{i}^{2}}\right)^{y_{i}^{r_{i, \ell}}}$ for all $\ell$.

$\Delta$ finally contains $g^{m}$, the $g^{m_{i}} \mathrm{~s}$, the $r_{i, \ell} \mathrm{s}$, the $\delta_{i, \ell} \mathrm{s}$, the $\gamma_{i, \ell} \mathrm{s}$, and $A_{j}$.

Reconstruction stage. Each participant $P_{i}$ decrypts his own share $m_{i}$ by computing

$$
m_{i}=\frac{\left(\sigma_{i}^{1}\right)^{z_{i}}}{\sigma_{i}^{2}} \bmod p .
$$

A subset of $k$ participants can then recover $m$ by using the Lagrange's interpolation formula.

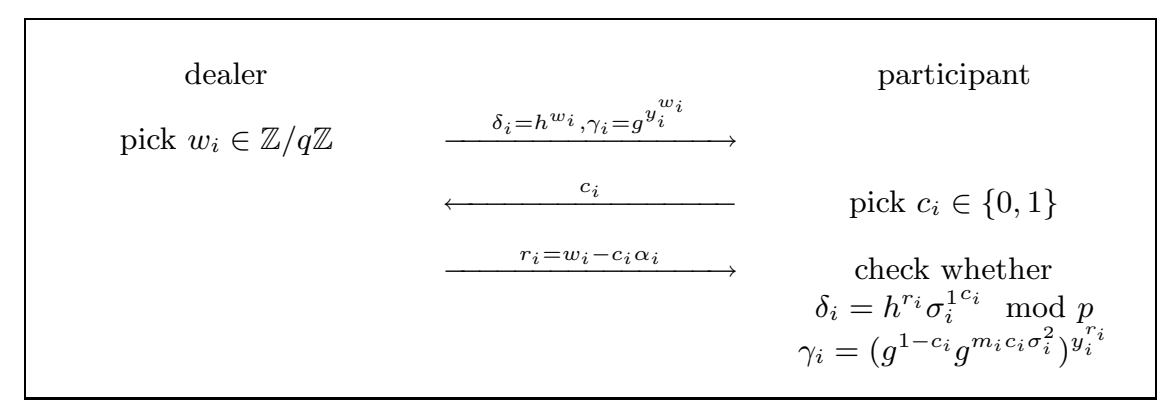

Fig. 1. Interactive verification procedure

\subsection{Primitives}

We define in this section some primitives which will be used in our protocol. These primitives are not related to a particular PVSS.

Signature. We consider $P_{o}$ 's authentication function $S_{o}$ which, given a message $m$, outputs the signed version $m^{\prime}=S_{o}(m)$ and the corresponding verification function $V_{o}$. Note that $S_{o}$ is either a signature with message recovery or the concatenation of the message with the signature.

Item description. As is typically assumed in the literature, we suppose that $P_{o}$ and $P_{r}$ have committed to their items beforehand. We consider therefore that $P_{o}$ and $P_{r}$ established a legal agreement linking the authentic human-readable descriptions of the items with mathematical descriptions of these items $\operatorname{descr}\left(m_{o}\right)$ and $\operatorname{descr}\left(m_{r}\right)$. For instance, $\operatorname{descr}(m)=\mathcal{W}(m)=g^{m}$. According to the fact that the authentic descriptions match the mathematical descriptions (a conflict at this layer can only be resolved by legal means), participants will be satisfied if they 
receive an item $m$ which is consistent with its description $\operatorname{descr}(m)$. To check that, we consider the public contract $\Omega=S_{o}\left(P_{o}\left\|P_{r}\right\| \operatorname{descr}\left(m_{o}\right)\left\|\operatorname{descr}\left(m_{r}\right)\right\| T\right)$, where $T$ is the expiration date after what the exchange has to be considered as null and void.

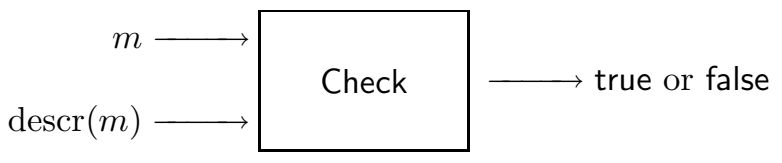

Encryption. We consider $P_{i}$ 's encryption function $E_{i}$ which, given a message $m$, outputs the encrypted message $m^{\prime}=E_{i}(m)$ for $P_{i}$ and the corresponding decryption function $D_{i}$ such that, given an encrypted message $m^{\prime}$, outputs the plain message $m=D_{i}\left(m^{\prime}\right)$.

Verifiable secret sharing. We consider a publicly verifiable secret sharing scheme using the functions Share, Verify, and Recover. Given a message $m$ and some participants $P_{i}(1 \leq i \leq n)$, Share outputs the encrypted shares $E_{1}\left(m_{1}\right), \ldots$, $E_{n}\left(m_{n}\right)$, and the proof $\Delta$, as described in Section 3.2; given a list of encrypted shares, a list of participants, $\Delta$, and $\operatorname{descr}(m)$, Verify outputs true if the shares allow any subset of $k$ participants to recover $m$ and false otherwise; given some shares $m_{i_{1}}, \ldots, m_{i_{k}}$ and participants $P_{i}\left(i \in\left\{i_{1}, \ldots, i_{k}\right\}\right)$, Recover outputs the message $m$.

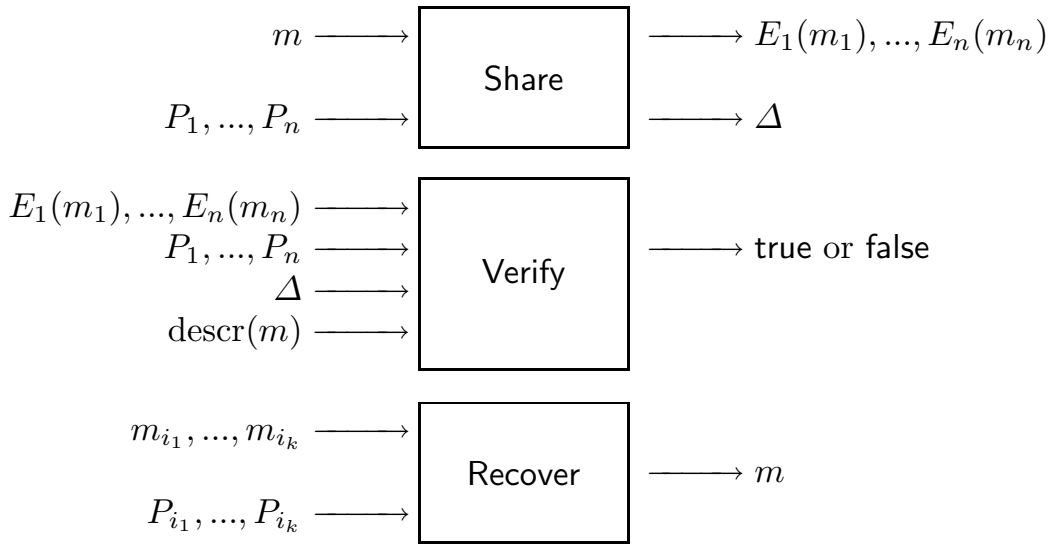

Verifiable encryption. We describe the CheckEnc function which is pretty similar to the Check function except that its input is $E_{o}(m)$ rather than $m$. This function is used by the passive participants. We will not detail this function for the sake of simplicity but it could come from a PVSS with a single participant. We only sketch the primitives related to CheckEnc:

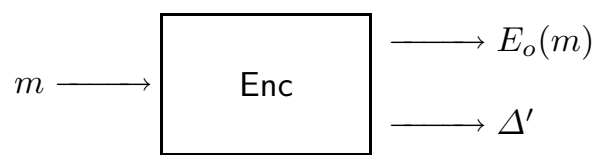




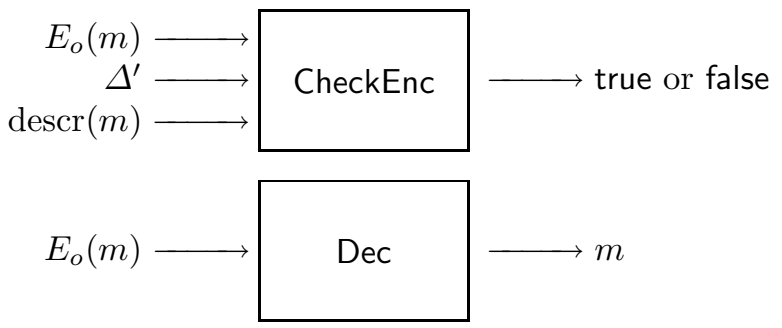

\subsection{Description of the Protocols}

Two participants $P_{o}$ and $P_{r}$ wish to exchange items $m_{o}$ and $m_{r}$ in a set of participants $\mathcal{P}$ such that $\mathcal{P} \subset E_{n v_{P_{o}}} \cap E_{n v_{P_{r}}}$ with $|\mathcal{P}|=n$. Note that for the sake of simplicity, we exclude $P_{o}$ and $P_{r}$ from $\mathcal{P}$, although it is not mandatory; so $\mathcal{P}$ contains only passive participants. Here are the exchange protocol and the recovery protocol.

Exchange protocol. The exchange protocol (Fig. 2) implies only the active participants, $P_{o}$ and $P_{r}$, and consists in exchanging items $m_{o}$ and $m_{r}$ after a commitment step. This commitment gives to $P_{r}$ the ability to restore fairness of the exchange, helped by the passive participants, in case of conflict with $P_{o}$.

- Step 1: $P_{o}$ picks a random element $a$ and computes $b$ such that $m_{o}=a+b$. He computes Share $\left(a, P_{1}, \ldots, P_{n}\right)$ and sends $E_{1}\left(a_{1}\right), \ldots, E_{n}\left(a_{n}\right), \Delta, \Omega, b$ to $P_{r}$.

- Step 2: $P_{r}$ checks that $\operatorname{Verify}\left(E_{1}\left(a_{1}\right), \ldots, E_{n}\left(a_{n}\right), P_{1}, \ldots, P_{n}, \Delta, \operatorname{descr}(a)\right)$ is true where $\operatorname{descr}(a)$ is deduced from $\operatorname{descr}\left(m_{o}\right)$ (extracted from $\Omega$ ) and $b$, e.g. $g^{a}=g^{m} \times g^{-b}$; if the test succeeds then he sends $m_{r}$ to $P_{o}$; otherwise he has just to wait until the expiration date $T$ to give up the exchange.

- Step 3: $P_{o}$ checks that $m_{r}$ is correct running $\operatorname{Check}\left(m_{r}, \operatorname{descr}\left(m_{r}\right)\right)$. If it is the case then $P_{o}$ sends $m_{o}$ to $P_{r}$. Otherwise, he has just to wait until the expiration date $T$ in order to give up the exchange.

- Step 4: If $P_{r}$ does not receive $m_{o}$ or if $\operatorname{Check}\left(m_{o}, \operatorname{descr}\left(m_{o}\right)\right)$ is false then he runs the recovery protocol.

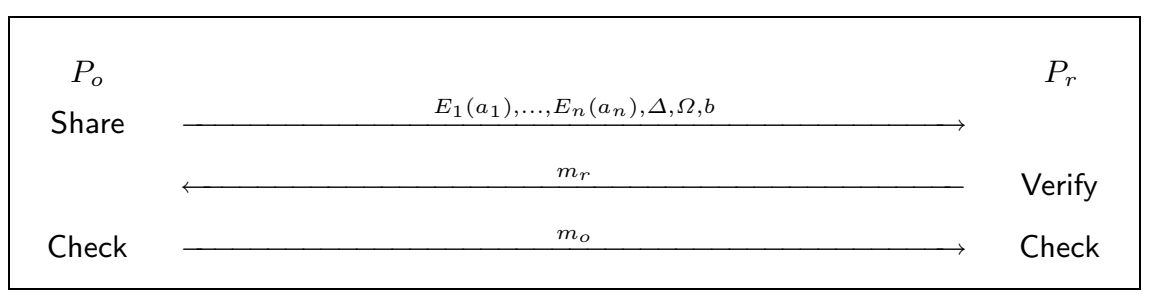

Fig. 2. exchange protocol 
Recovery protocol. The recovery protocol (Fig. 3) is started before $T-T_{\max }$ by the recipient, $P_{r}$, when he is injured, that is if the third message of the exchange, $m_{o}$, is wrong or missing.

- Step 1: $P_{r}$ encrypts $m_{r}$ for $P_{o}$ and sends $E_{i}\left(a_{i}\right), E_{o}\left(m_{r}\right), \Delta^{\prime}$, and $\Omega$ to $P_{i}$.

- Step 2: $P_{i}$ computes CheckEnc $\left(E_{o}\left(m_{r}\right), \operatorname{descr}\left(m_{r}\right), \Delta^{\prime}\right)$ where $\operatorname{descr}\left(m_{r}\right)$ is extracted from $\Omega$; if the output is true and if the expiration date, contained in $\Omega$, has not expired, $P_{i}$ sends $a_{i}$ to $P_{r}$ and $E_{o}\left(m_{r}\right)$ to $P_{o}$.

- Step 3: after having received $k$ shares, $P_{r}$ runs Recover. From $a$ he computes $m_{o}=a+b$.

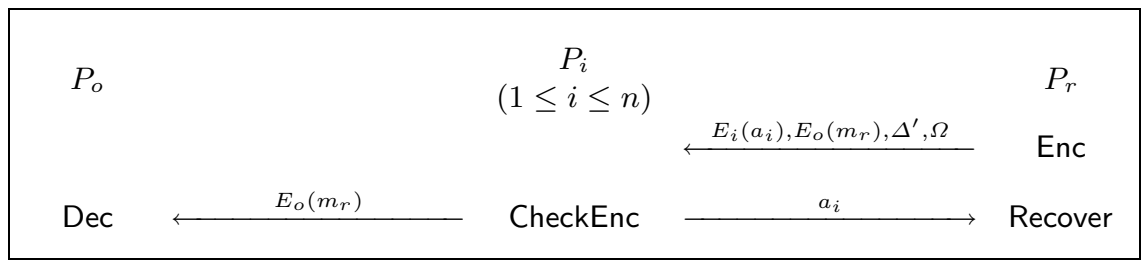

Fig. 3. recovery protocol

Remark 4. In optimistic fair exchange protocols using TTP (e.g. [11]), the TTP is stateful: the TTP keeps in mind whether the recovery or abort protocol has already been performed. Due to our distributed architecture we cannot use this model here and we prefer using expiration dates.

\section{Security Analysis}

We prove in this section that our protocol is complete, fair, timely and respects the privacy property even in case of misbehaviors. We recall that the security parameter $k$, which is the threshold of the PVSS, is such that $b_{2}<k \leq b_{2}+b_{1}$. We defined in Section 2.2 the set of passive participants $\mathcal{B}_{1}, \mathcal{B}_{2}, \mathcal{B}_{3}$, and $\mathcal{B}_{4}$. We rewrite these definitions here according to our protocol defined in Section 3.4: $\mathcal{B}_{1}$ : participants who honestly collaborate with both $P_{o}$ and $P_{r} ; \mathcal{B}_{2}$ : participants $P_{i}$ such that when $P_{r}$ sends $E_{i}\left(a_{i}\right)$ to $P_{i} \in \mathcal{B}_{2}, P_{i}$ decrypts $E_{i}\left(a_{i}\right)$ and sends $a_{i}$ to $P_{r}$ (even if the date is expired) but does not send $m_{r}$ to $P_{o} ; \mathcal{B}_{3}$ : participants $P_{i}$ such that when $P_{r}$ sends $E_{i}\left(a_{i}\right)$ to $P_{i} \in \mathcal{B}_{3}, P_{i}$ sends $E_{o}\left(m_{r}\right)$ to $P_{o}$ (even if the date is expired) but does not decrypt $E_{i}\left(a_{i}\right)$ for $P_{r} ; \mathcal{B}_{4}$ : participants who do not collaborate at all. We denote $M_{1}, M_{2}$, and $M_{3}$ the three messages of the exchange protocol consisting respectively of $\left[E_{1}\left(a_{1}\right), \ldots, E_{n}\left(a_{n}\right), \Delta, \Omega, b\right],\left[m_{r}\right]$, and $\left[m_{o}\right]$. 


\subsection{Completeness of the Protocol}

Proving that the protocol is complete when $P_{o}$ and $P_{r}$ are honest is straightforward. In case of late discovery of $M_{3}$ due to communication protocol reasons, $P_{r}$ runs the recovery protocol. Since $b_{2}<k \leq b_{2}+b_{1}$ we have at least $k$ participants who will collaborate with $P_{r}$ and at least one who will collaborate with $P_{o}$.

\subsection{Fairness of the Protocol}

We saw in the previous section that the protocol is fair if both active participants are honest. We explained furthermore in Section 2.2 that the case where both active participants are dishonest is not relevant.

$P_{o}$ is honest and $P_{r}$ is dishonest. Since $P_{o}$ is honest, $M_{1}$ is correctly formed. On the other hand $M_{2}$ is wrong (or missing) otherwise both $P_{o}$ and $P_{r}$ would be honest. Here $P_{o}$ can detect that $M_{2}$ is wrong using $\operatorname{Check}\left(m_{r}, \operatorname{descr}\left(m_{r}\right)\right)$; therefore he does not transmit $M_{3}$ and waits for $T$. If $P_{r}$ does not run the recovery protocol then nobody can obtain anything valuable on the expected items and the exchange is trivially fair. If $P_{r}$ starts the recovery protocol after $T$ then he cannot obtain $m_{o}$ since $b_{2}<k$. If $P_{r}$ starts the recovery protocol before $T$ (note that if he only contacts participants in $\mathcal{B}_{2}$ or $\mathcal{B}_{4}$ before $T$ then we fall into the previous case) then $P_{o}$ receives $m_{r}$ from a passive participant either in $\mathcal{B}_{1}$ or $\mathcal{B}_{3}$; therefore the protocol is fair iff $P_{r}$ can obtain $m_{o}$ that is if and only if $b_{1}+b_{2} \geq k$.

$P_{o}$ is dishonest and $P_{r}$ is honest. Since $P_{o}$ is dishonest, we consider the case where $M_{1}$ is wrong (or missing) and the case where $M_{1}$ is correct but $M_{3}$ is not (or missing). If $M_{1}$ is wrong (or missing), the exchange will die after $T$; indeed $P_{r}$ can perform Verify $\left(E_{1}\left(a_{1}\right), \ldots, E_{n}\left(a_{n}\right), P_{1}, \ldots, P_{n}, \Delta, \operatorname{descr}(a)\right)$ and detects so that $M_{1}$ is wrong; he decides therefore not to disclose $m_{r}$. The exchange ends therefore on a trivially fair termination after $T$. Secondly, if $M_{1}$ is correct but $M_{3}$ is not (or missing): $P_{r}$ can detect such a wrong $M_{3}$ using Check $\left(m_{o}, \operatorname{descr}\left(m_{0}\right)\right)$ and therefore start the recovery protocol. The fairness of the exchange relies thus on the ability of the passive participant to supply $a$ to $P_{r}$, that is if and only if $b_{1}+b_{2} \geq k$. The fairness is so ensured since $P_{o}$ has already received $m_{r}$ in $M_{2}$.

\subsection{Timeliness of the Protocol}

Timeliness of the protocol is straightforward.

\subsection{Privacy of the Protocol}

Privacy of the protocol is straightforward. If the recovery protocol is not performed, then only information between $P_{o}$ and $P_{r}$ are exchanged and passive participants receive nothing. If the recovery protocol is used, then some participants receive shares of $a_{i}$. However, although $k$ participants colluding can recover $a$, they cannot recover $m_{o}$ since they do not know $b$. Obviously, they 
cannot discover $m_{r}$ either. Privacy is here a great improvement with regard to previous optimistic fair exchange protocol where we usually assume that the trusted third party is able to regenerate expected items.

\subsection{Complexity of the Protocol}

When both $P_{o}$ and $P_{r}$ are honest, the complexity in terms of exchanged messages is very small since only the three messages of the exchange protocol are sent. When somebody misbehaves, the complexity obviously increases since the recovery procedure is performed. In the worst case, the $n$ passive participants are contacted by $P_{r}$, each receives one message and sends at most two messages, so the complexity is only $O(3 n)$ in terms of exchanged messages.

\section{Conclusion}

We proposed an optimistic two-party fair exchange protocol using publicly verifiable secret sharing. Our protocol is the first optimistic fair exchange protocol which does not rely on a centralized trusted third party. This concept is therefore particularly suitable for ad-hoc networks. We proved that our protocol ensures fairness and privacy even in quite dishonest environment and implies only low communication overheads. Our protocol works assuming that a majority of participants are honest or that only one is honest but we can estimate the number $b_{2}$ of participants who may harm $P_{o}$ by colluding with $P_{r}$.

\section{Acknowledgment}

The work presented in this paper was supported (in part) by the National Competence Center in Research on Mobile Information and Communication Systems (NCCR-MICS), a center supported by the Swiss National Science Foundation under grant number 5005-67322.

\section{References}

1. N. Asokan, Matthias Schunter, and Michael Waidner. Optimistic protocols for fair exchange. In Proceedings of 4th ACM Conference on Computer and Communications Security, pages 7-17, Zurich, Switzerland, April 1997. ACM Press.

2. N. Asokan, Victor Shoup, and Michael Waidner. Asynchronous protocols for optimistic fair exchange. In Proceedings of the IEEE Symposium on Research in Security and Privacy, pages 86-99, Oakland, California, USA, May 1998. IEEE Computer Society Press.

3. Gildas Avoine and Serge Vaudenay. Fair exchange with guardian angels. In Kijoon Chae and Moti Yung, editors, The 4th International Workshop on Information Security Applications - WISA 2003, volume 2908 of Lecture Notes in Computer Science, pages 188-202, Jeju Island, Korea, August 2003. Springer-Verlag. 
4. George Robert Blakley. Safeguarding cryptographic keys. In National Computer Conference, volume 48 of American Federation of Information, pages 313-317, 1979.

5. Ernest F. Brickell, David Chaum, Ivan B. Damgård, and Jeroen van de Graaf. Gradual and verifiable release of a secret. In Carl Pomerance, editor, Advances in Cryptology - CRYPTO'87, volume 293 of Lecture Notes in Computer Science, pages 156-166, Santa Barbara, CA, USA, August 1988. IACR, Springer-Verlag.

6. Benny Chor, Shafi Goldwasser, Silvio Micali, and Baruch Awerbuch. Verifiable secret sharing and achieving simultaneity in the presence of faults. In Proceedings of the 26th IEEE Annual Symposium on Foundations of Computer Science FOCS'85, pages 383- 395, Portland, OR, USA, October 1985. IEEE.

7. Richard Cleve. Controlled gradual disclosure schemes for random bits and their applications. In Gilles Brassard, editor, Advances in Cryptology - CRYPTO'89, volume 435 of Lecture Notes in Computer Science, pages 573-588, Santa Barbara, California, USA, August 1990. IACR, Springer-Verlag.

8. Taher El Gamal. A public key cryptosystem and a signature scheme based on discrete logarithms. IEEE Transactions on Information Theory, 31(4):469-472, July 1985.

9. Shimon Even and Yacov Yacobi. Relations amoung public key signature systems. Technical Report 175, Computer Science Department, Technicon, Haifa, Israel, 1980 .

10. Eiichiro Fujisaki and Tatsuaki Okamoto. A practical and provably secure scheme for publicly veriable secret sharing and its applications. In Nyberg Kaisa, editor, Advances in Cryptology - EUROCRYPT'98, volume 1403 of Lecture Notes in Computer Science, pages 32-46, Helsinki, Finland, May-June 1998. IACR, SpringerVerlag.

11. Olivier Markowitch and Shahrokh Saeednia. Optimistic fair exchange with transparent signature recovery. In Financial Cryptography - FC'01, Lecture Notes in Computer Science, Cayman Islands, February 2001. IFCA, Springer-Verlag.

12. Berry Schoenmakers. A simple publicly verifiable secret sharing scheme and its application to electronic voting. In Michael Wiener, editor, Advances in Cryptology - CRYPTO'99, volume 1666 of Lecture Notes in Computer Science, pages 148-164, Santa Barbara, California, USA, August 1999. IACR, Springer-Verlag.

13. Adi Shamir. How to share a secret. Communications of the ACM, 22(11):612-613, November 1979.

14. Markus Stadler. Publicly verifiable secret sharing. In Ueli Maurer, editor, Advances in Cryptology - EUROCRYPT'96, volume 1070 of Lecture Notes in Computer Science, pages 190-199, Saragossa, Spain, May 1996. IACR, Springer-Verlag.

15. Holger Vogt. Asynchronous optimistic fair exchange based on revocable items. In Rebecca N. Wright, editor, Financial Cryptography - FC'03, volume 2742 of Lecture Notes in Computer Science, Le Gosier, Guadeloupe, French West Indies, January 2003. IFCA, Springer-Verlag. 